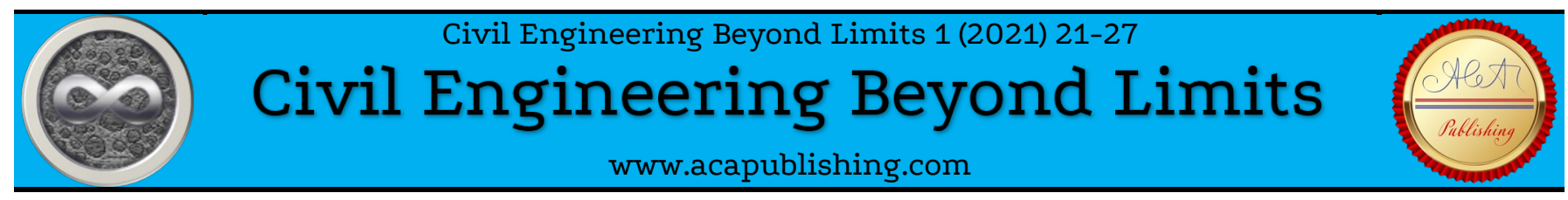

Research Article

\title{
Preliminary Finite Element Modeling of Asphalt Material Using ANSYS
}

\author{
Nitish R. Bastola ${ }^{1}$, Mena I. Souliman ${ }^{2}$, and Zabi Ahmed Mohammed ${ }^{1}$ \\ ${ }^{1}$ Graduate Research Assistant, The University of Texas at Tyler; \\ ${ }^{2}$ Associate Professor, The University of Texas at Tyler.
}

\section{Keywords}

Finite Element Modeling, Asphalt, Fatigue Test, Four-Point Bending Test, Pavement Materials

\begin{abstract}
Finite element modeling (FEM) has been an integral tool for civil engineering researchers. The simplification of day to day engineering problems with known scientific validation makes this tool more appropriate. In the field of pavement engineering, the use of FEM has been a relatively new tool. Various conditions of pavement materials, which are itself complex in nature, makes the modeling more challenging. This study aims to develop a basic beam model as per AASHTO T321 for evaluating the performance of asphalt material under fatigue loading. The necessary conditions and parameters are best optimized to match the research on National Cooperative Highway Research Program Project 9-44A (NCHRP Report 762). Various mechanical and fatigue-related properties of asphalt materials were studied to develop a simplified finite element approach through ANSYS. It is observed that stiffness induced behavior and fatigue performance can be predicted in advance and in a timely fashion with the utilization of FEM when compared to performing actual laboratory testing. In this study, different life and damage analyses were performed for different sets of the beam. It was found that stiffness plays a significant role in enhancing the minimum critical life of asphalt materials. A higher stiffness of the asphalt beam characterized a higher minimum critical life, and lower stiffness characterized the lower minimum critical life.
\end{abstract}

\begin{abstract}
1. Introduction
In the field of designing and analysis, finite element modeling (FEM) has been an integral tool. Many experimental designs to be performed in the laboratory setup can be completed effortlessly with the help of FEM. FEM has also been a reliable tool for the early estimation of different physical systems and processes. Various research studies are being performed in the field of pavement engineering with the utilization of FEM, even though the applications are minimum. The utilization is mostly over the failures characterization.
\end{abstract}

Pavement structure failures are characterized by various kinds of distresses. Among them, fatigue failure is referred to as one of the critical forms of distress. Developed due to the excessive repetition of wheel loads, fatigue failure is always a matter of concern among the researchers and engineers. The property of asphalt being utilized plays a crucial role in the fatigue-free performance of the pavement. Early performance estimation of the pavement structure makes the designer aware of the choice of material types. American Association of State Highway Transportation Officials (AASHTO) provides clear guidance about the experimental analysis of fatigue on asphalt mixture samples through the reference T321. Experimental perspectives for the fatigue test utilizing 4 point bending apparatus are the parts of the manual. In this study, the same conditions of judging the asphalt materials as described by T321 are made to achieve an easier and quicker way through finite element simulation. Three independent asphalt beams are considered for the analysis based on research by Souliman (2012) [1] and NCHRP report 762 under National Cooperative Highway Research Program Project 9-44A (2013) [2]. Eventually, fatigue performances of the beams are evaluated concerning fatigue cycles of the beam.

\section{Literature Review}

The importance of finite element modeling on a day to day engineering and scientific works was already discussed in the earlier section. The nature of these works is advanced since early knowledge of the working mechanism is required. Various research have been performed to date in the field of FEM and these research projects are broadly practiced in various fields of engineering. Moreover, in pavement engineering, the use of finite element analysis (FEA) is a new concept, although some profound research has been done utilizing various tests, including the 4-point bending test.

There are various tools for FEA of the pavement structures. ABAQUS and ANSYS are renowned software in the field. Although ABAQUS is popular among researchers, this study tries to explore ANSYS to find its applicability in the field of pavement engineering. Numerous literature reviews for FEM in the pavement, along with a 4-point bending test have been performed to grasp the knowledge about ANSYS and its applicability.

\subsection{Finite Element Modelling in Pavement Engineering}

A pavement structure is composed of various layers including subbase, base, and Hot mixed aphalt (HMA) surface layers. The properties of these layers are material dependent; therefore, understanding the material's properties is vital for FEM. Sukumaran et al. (2005) [3] performed a preliminary investigation on 3D modeling of pavement structures. In this study, FEM of flexible pavement was utilized in the airport with the guidance of the National Pavement Test Facility. The data required for the study was from the facility itself, and the finite element system developed in this study was utilized in determining the failure mechanism of pavement surface under moving aircraft loads.

Similarly, another research is performed for the asphalt concrete response simulation through the utilization of the finite element 
model. Erkens et al. (2006)[4]performed this study intending to formulate temperature, loading history-dependent, and strain rate sensitive three-dimensional model. The model parameters were developed through experimental setups and testing. Experimental testing, as well as the model verification were paralleled throughout the project. The initiation and propagation of the damage in two flexible pavement structures have been simulated in this study. The influence of geometry and material characteristics were studied to know the damages.

A study conducted by Hadi and Bodhinayake (2003) [5]incorporates the moving traffic load and the realistic mechanical properties of the pavement layers following the finite element theory. A pavement structure with cyclic loading simulation is obtained and analyzed as a finite element model. Various static and cyclic loading are considered in the study. Eventually, the results are compared, and it was found that the cyclic loading with the non-linear materials pertains to the most similar case scenario to field measured deflections [5].

In another study dealing with the semi-analytical finite element method for judging the bearing capacity of asphalt pavement, Liu et al. (2015)[6] utilized FEA in developing software in the analysis of asphalt pavement. The main objective for the development of the analysis was overcoming the difficulty of real traffic loading conditions. The investigation proved to be a feasible option for the swift and reliable analysis of pavement bearing capacity when the finite element approach was utilized at the network level of road systems.

\subsection{Utilization of ANSYS in Finite Element Modelling of Pavement}

Research by Nilaward et al. (1998)[7], under the Federal Highway Research Program was based on the development of a 3-dimensional program for the analysis of the pavement systems. The utilization of the explicit approach and the vector formulation of the equation of motion were studied. A two-dimensional finite element program was also developed for the convenience of application. Various loading conditions were verified with pulse input, prescribed ground acceleration, static ramp, step loadings, and sinusoidal loadings. The verification procedure was performed with ANSYS, where material library-related verifications were performed for elastic-plastic materials with Mises criteria in the assumption of associated and non-associated flow rules and a viscoelastic material of Maxwell Type.

Similarly, response analysis of flexible pavement utilizing ANSYS was performed by Mulungye et al. (2005) [8].In this study, a finite element viscoelasticity model was implemented to predict the performance of a pavement structure. Delayed lateral strain relaxation under traffic loads was a prime objective of the utilization of FEM. The fatigue life of the pavement structure was determined in this study.

Vijapure et al. (2005)[9]also performed a FEA of flexible pavement. In this study, mechanistic design techniques are explored to determine stress, strain, and displacement in the pavement analysis. Ultimately, a 3D finite element application is utilized for the prediction of mechanical behavior and the pavement's performance under varying traffic loads.

\subsection{Fatigue Analysis of Asphalt Beam: Experimental and Finite Element Approach}

The four-point bending test, also referred as the beam fatigue test, is one of the widely utilized tests in determining the fatigue life of an asphalt beam under repeated loads. Various researchers have numerous studies and theories in the utilization of experimental and finite element approach through a four-point bending test. The following figure presents a 4-point bending device with the HMA specimen.

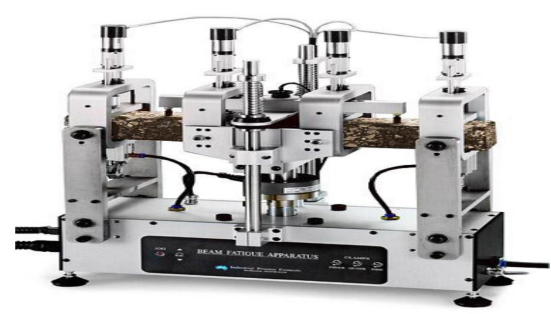

Figure 1. 4 Point Bending Device [1]

Souliman (2012) [1] performed the fatigue study of the asphalt beam in the laboratory to determine the fatigue endurance limit of asphalt pavement. 468 fatigue beam tests were performed in the laboratory setting. Various conclusions related to endurance limit, strain conditions, and failure cycle were emphasized in the study.

Another study by Adhikari and You (2005)[10]performed the fatigue evaluation of asphalt pavement using a beam fatigue apparatus. In this study, a typical Michigan asphalt mixture was utilized under various temperature and frequencies. Evaluation of different fatigue performance models under various laboratory testing conditions was achieved. The study shows a linkage between compression modulus and flexural stiffness. Rutting and fatigue due to vertical compression and flexural bending respectively are conceptualized with the help of compression modulus.

In a study, Coni et al. (2008) [11] performed the finite element analysis of the 4-point bending test for fatigue cracking. The fatigue life of an asphalt mixture was evaluated based on factors such as loading conditions, testing setup, and internal stress or strains imposed by the clamps at the load and reaction points. Framing of the beam, structure, and geometry was designed in Nottingham Transportation Center (NTEC). The imprecision of the test results and consideration of it in design and optimization are discussed in the study.

A study by Huurman and Pronk (2012) [12]demonstrated the detailed FEA of the 4-point bending test device. The study provides insight into the modeling and working of the devices associated with fatigue cases. Similarly, calibration related factors between the actual devices and the modeled device were also coined in the study.

The extensive literature review presented above was utilized to understand the necessary conditions required for the FEA of asphalt materials. The research studies were utilized in evaluating how the modeling was performed by various researchers. Exploration of ANSYS as a useful tool in pavement engineering was made on the research presented in the literature review. Therefore, these literature reviews provide a backbone for analyzing asphalt materials through a simplified procedure of a 4-point bending beam fatigue test. Various conditions necessary for judging asphalt pavement are presented in the proceeding sections.

\section{Objectives}

The research aims to utilize the finite element approach in the analysis of asphalt materials through a well-known 4-point bending beam fatigue test. Various performance evaluations of the asphalt material, including fatigue life and damage, were observed. Eventually, a simplified method is formulated as an early procedure of predicting the materialistic fatigue performance of the asphalt pavement.

\section{4-Point Beam Test of Asphalt Materials}

A 4-point bending test is one of the essential tests for characterizing the properties of an asphalt material. Fatigue, flexural characteristics can be easily analyzed through the help of a 4-point bending beam test and the materials which are weak in shear loadings are often provided with a 4-point bending test. Asphalt materials being 
relatively weak in shear are loaded with this kind of loadings. This section of the study provides a brief background on the ANSYS related four-point bending test and various procedures followed. Each of the procedures followed is described briefly in the following sub-heading.

\subsection{Data collection Necessary for Modelling}

The necessary data utilized in this study is obtained from the research performed by Souliman (2012) [1]. The study was purely based on the experimental analysis of the beam fatigue test. The beam dimensions, stiffness parameters, as well as other necessary inputs, were synchronized following the same study. Table 1 shows the stiffness value considered in the study.

Table 1. Stiffness Value Considered in the Study

\begin{tabular}{ccc}
\hline Beam Sp. & Stiffness & $\begin{array}{c}\text { Stiffness Value } \\
(\mathrm{MPa})\end{array}$ \\
\hline a & High & 15436.68 \\
b & Medium & 7672.49 \\
c & Low & 234.77 \\
\hline
\end{tabular}

Similarly, to characterize the viscoelastic nature of the asphalt beam, Table 2, with references from the research study by Mulungye et al. (2005) [8], is fitted in ANSYS. Figure 2 shows the data utilized with the performance curve (ANSYS Generated).

Table 2. Viscoelastic Curve Fitting Data for Fatigue Test [8]

\begin{tabular}{|c|c|c|}
\hline No. of Cycles & Time (s) & Alternating Stress (MPa) \\
\hline 10 & 2.5 & 2536 \\
\hline 20 & 5 & 2383 \\
\hline 30 & 7.5 & 2440 \\
\hline 40 & 10 & 2437 \\
\hline 50 & 12.5 & 2469 \\
\hline 60 & 15 & 2495 \\
\hline 70 & 17.5 & 2561 \\
\hline 80 & 20 & 2528 \\
\hline 90 & 22.5 & 2475 \\
\hline 100 & 25 & 2392 \\
\hline 200 & 50 & 1550 \\
\hline 300 & 75 & 1521 \\
\hline 400 & 100 & 1551 \\
\hline 500 & 125 & 1563 \\
\hline 550 & 137.5 & 1601 \\
\hline 950 & 237.5 & 1402 \\
\hline 1050 & 262.5 & 1390 \\
\hline 1150 & 287.5 & 1408 \\
\hline 1250 & 312.5 & 1420 \\
\hline 1350 & 337.5 & 1462 \\
\hline 1450 & 362.5 & 1481 \\
\hline 1550 & 387.5 & 1474 \\
\hline 2550 & 637.5 & 1298 \\
\hline 3550 & 887.5 & 1043 \\
\hline 4550 & 1137.5 & 589 \\
\hline 4590 & 1147.5 & 551 \\
\hline
\end{tabular}
2012. Through this study, beam size and the properties associated were all collected. The following Figure 3 shows the dimensions of the beam used in the study.

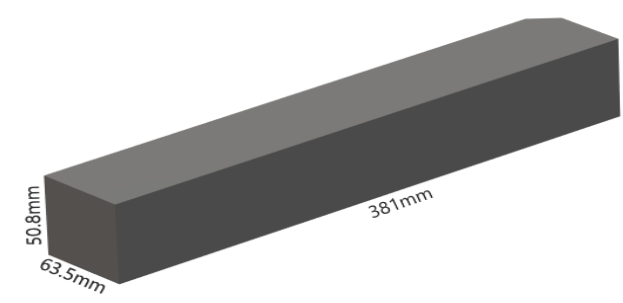

Figure 3. Standard Beam with Dimensions

With the given beam, load plates (each $5 \mathrm{~mm}$ thick and $25 \mathrm{~mm}$ in length) were assigned at four corners. Similar load plates were also assigned at one-third of the beam at four different places to simulate the actual flexural apparatus. Figure 4, presented below, is the modeled beam with load plates and meshing.

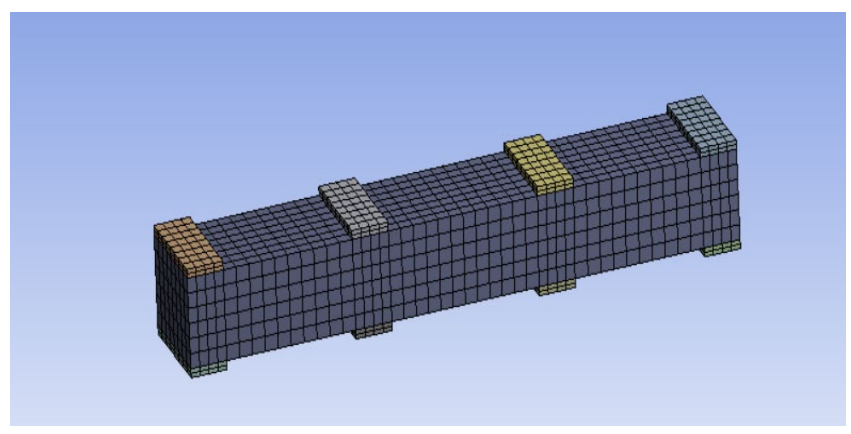

Figure 4. Meshed Beam with Load Plates 


\subsection{Assignment of the Materials}

Steel and asphalt materials were utilized in this study. The beam utilized in the analysis is made up of asphalt materials with a density of $2400 \mathrm{~kg} / \mathrm{m} 3$, and the steel plates are assigned as loading plates. The density of steel is $7850 \mathrm{~kg} / \mathrm{m} 3$. The Poisson's ratio and the other material properties were assigned following derivation from the modulus.

\subsection{Assignment of Contact Surface, Loads, and Necessary Boundary Conditions}

With the assignment of the materials, the necessary conditions for the analysis are to be introduced in the structure. One of the essential procedures followed in the study is the assignment of the contact surface. ANSYS offers various forms of contact analysis, such as bonded, frictional, rough, and many others. In this study, bonded contact is assumed, and the further procedure is followed up. The following Figure 5 shows the target and contact in the structure.

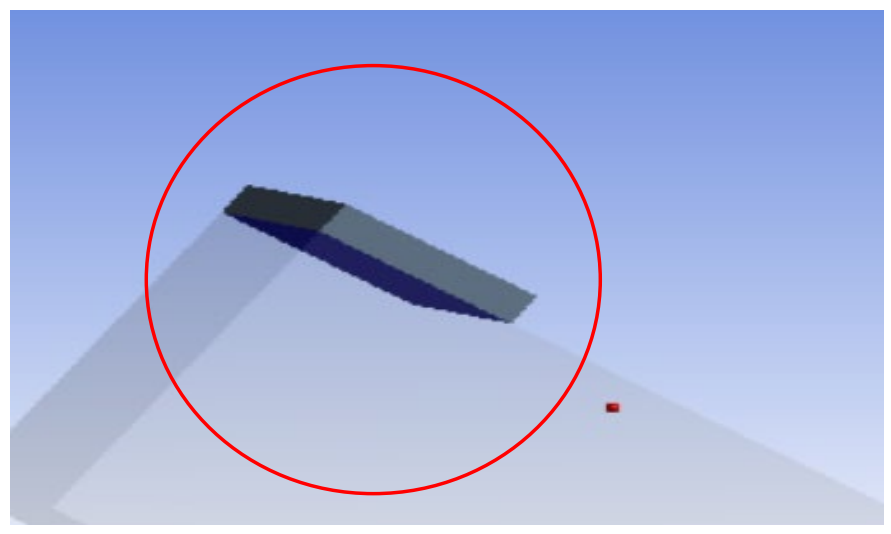

Figure 5. Contact in Between Beam and Plate

Two-point loads were applied on the steel plates provided at one-third of the beam. In order to make everything uniform, the loads were set at a constant value for all the cases. Similarly, the boundary conditions were set up as the simply supported beam for providing the conditions of the actual flexural testing machine. The following Figure 6 shows the necessary load setup and boundary conditions implemented.

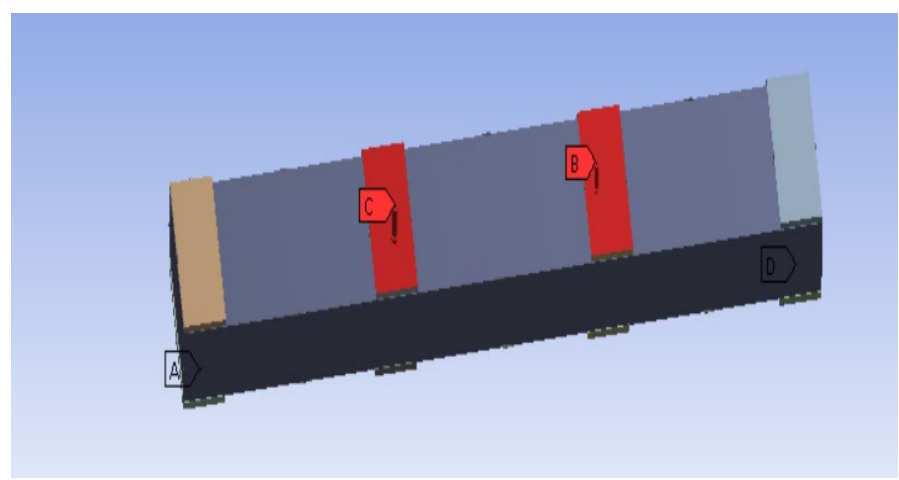

Figure 6. Beam with Boundary Conditions and Loads

\section{Analysis Through Simulated Asphalt Beam Fatigue Test}

The strategic analysis of the beam was conducted under various conditions of the stiffness value. These analyses were studied on various headings such as stress analysis, strain analysis, total deformation analysis, fatigue cycle, and life analysis.

\subsection{Total Deformation Analysis}

The total deformation obtained from the analysis is presented in Figure 7. The deformation was maximum and the maximum displacement deduced from analysis were both at the center of the beam. The maximum value is $0.005 \mathrm{~m}$. Similarly, the minimum deformation was at the edge of the beam with a value of 0 .

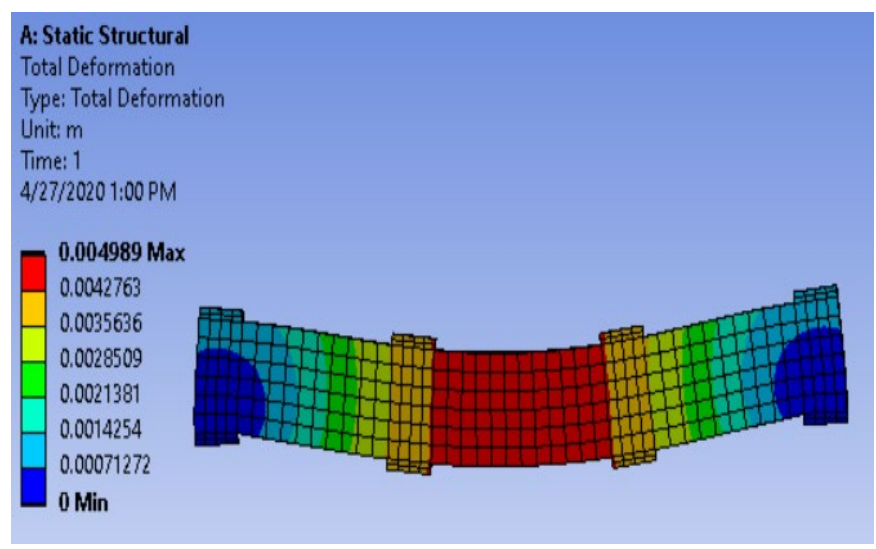

Figure 7. Total Deformation in Asphalt Beam Specification a

\subsection{Stress Analysis}

Stress analysis of any specimen is a valuable tool in knowing the performance of ductile materials. Based on the theory of fracture, von mises stress is utilized in the study, since the asphalt material is ductile to some extent. The following Figure 8 represents the stress and the performance of the asphalt beams in the case of given loadings. The beam has a higher stress concentration on the restraints.

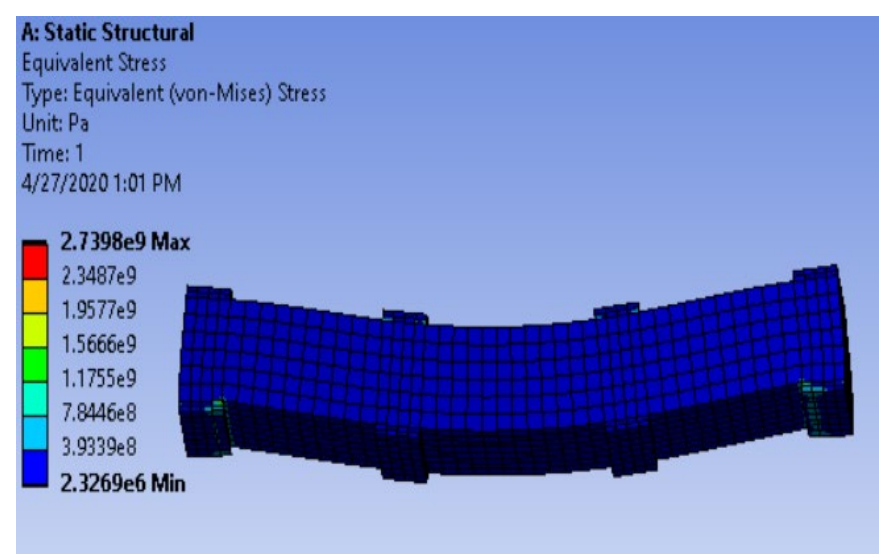

Figure 8. Stress Occurrence in Asphalt Beam Specification ab z

\subsection{Strain Analysis}

Similarly, strain analysis was performed for the given set of beams; the strain at various points of the beam is shown in the figure presented below. Figure 9 presents the result of the analysis. The maximum strain of $0.053 \mathrm{~m} / \mathrm{m}$ occurred at the beam. 


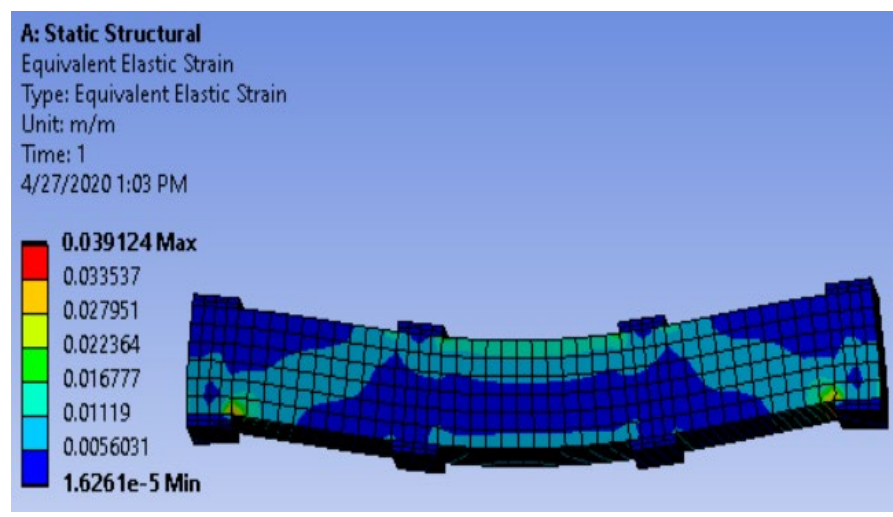

Figure 9. Strain Occurrence in Asphalt Beam Specification a

\subsection{Extensive Fatigue Analysis}

Fatigue analysis is one of the crucial procedures in material engineering, and they are specific in knowing the performance of under repeated loadings. Fatigues related results are often correlated for stress life, strain life, and crack growth. The materials with a long life or the one which withstands high cyclic failure, are studied through stress life. Similarly, strain life is utilized for the brittle materials, and the crack growth method is utilized for the material where the cracks prevail [13]. The following Figure 10 shows the fatigue life flow chart in analysis, which is similar to another form of analysis.

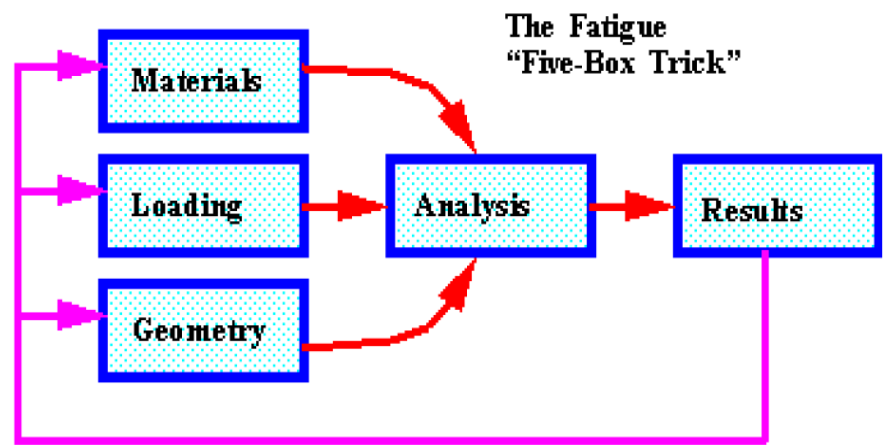

Figure 10. Fatigue Life Analysis Flow Chart [14]

In this study, various modes of fatigue analysis are explored concerning the fatigue life analysis and the fatigue damage analysis.The fatigue life analysis was formulated to know the life and damage in the material, respectively. To carry out this analysis, three different beam specimens, with high stiffness, medium stiffness, and low stiffness, were acquired from the research by Souliman (2012) [1]. The stiffness values for these beams were inputted in the ANSYS. The beams were selected in such a way that the following factors are similar.

\begin{tabular}{ll} 
- & Asphalt Content Percentage (AC \%) \\
- & Target Void Ratio (Va \%) \\
- & Applied Strain \\
\hline & Initial Stress
\end{tabular}

With these constraints, three set of beams were extracted out of the sets of 468 beams from the analysis by Souliman (2012) [1]. The beam specification and the stiffness are shown in table 1 under chapter 3.

The following Figures, 11 ( $a, b, c)$ show the beam specimens' performance under the fatigue life analysis. It is observed that the critical fatigue life is more significant for the beam with high stiffness than the one with the lesser stiffness.
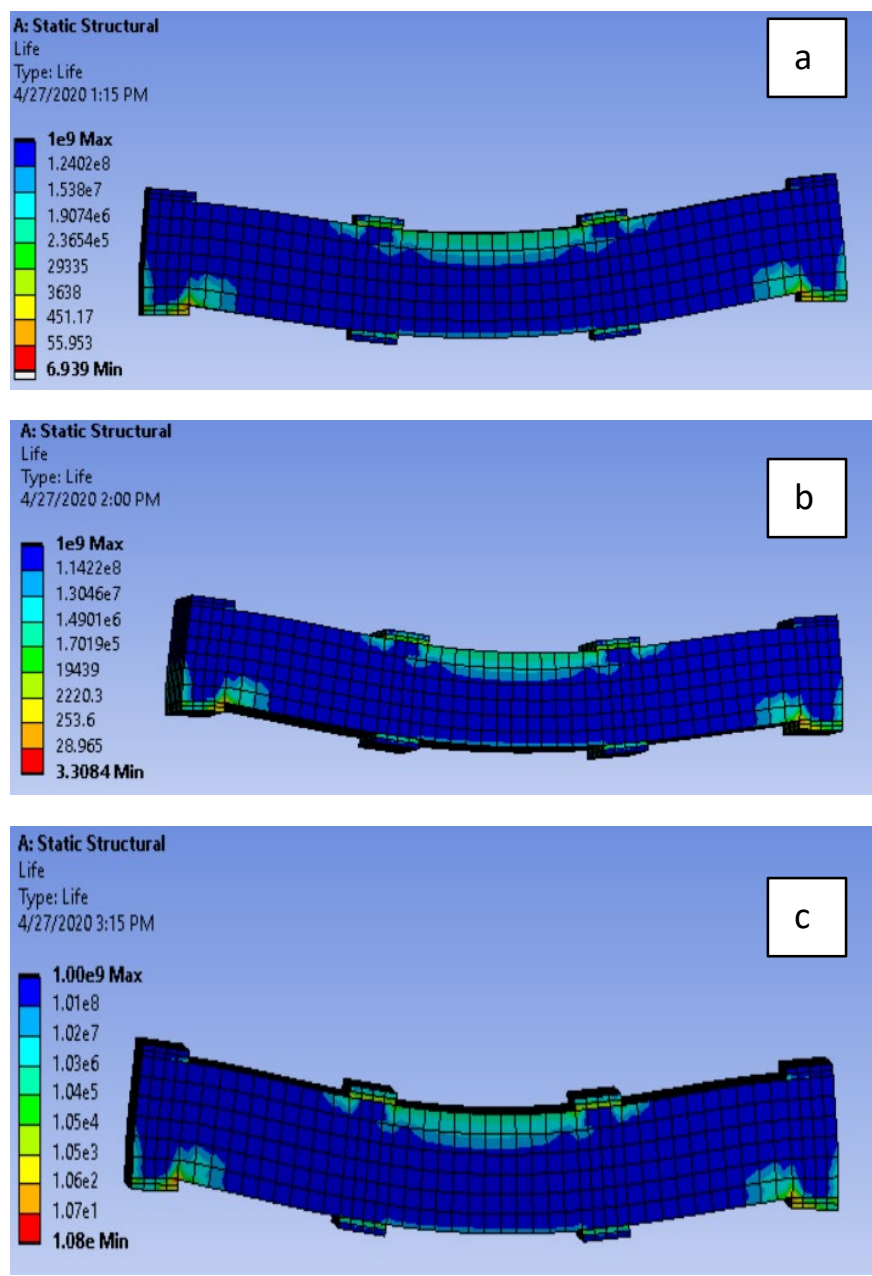

Figure 11. Fatigue Life Analysis of 3 Different Beam Specimens

Similarly, the damage related fatigue analysis of the beam in three cases are presented in the following figure 12 (a, b, c). The damage cycles are comparatively less for the beam with high stiffness than the one with less stiffness. The cycles required for the damage and failure of the beam specimen is tabulated in the figures representing the red as a maximum cycle it can withstand in its lifetime. The less stiffened beam can withstand more number of loading cycles compared to the beam which is highly stiffened.

Also, Table 3 summarizes the fatigue life and damages associated with the cases presented.

Table 3. Comparison of Various Fatigue Results

\begin{tabular}{cccc}
\hline Beam Sp. & $\begin{array}{c}\text { Stiffness } \\
(\mathrm{MPa})\end{array}$ & $\begin{array}{c}\text { Life Analysis } \\
\text { (Minimum } \\
\text { Critical Life) }\end{array}$ & $\begin{array}{c}\text { Damage } \\
\text { Analysis } \\
\text { (Cycles before } \\
\text { Failure 10^8) }\end{array}$ \\
\hline $\mathrm{a}$ & 15436.68 & 6.939 & 1.441 \\
$\mathrm{~b}$ & 7672.49 & 3.3 & 3.022 \\
$\mathrm{c}$ & 234.77 & 1.08 & 9.237 \\
\hline
\end{tabular}



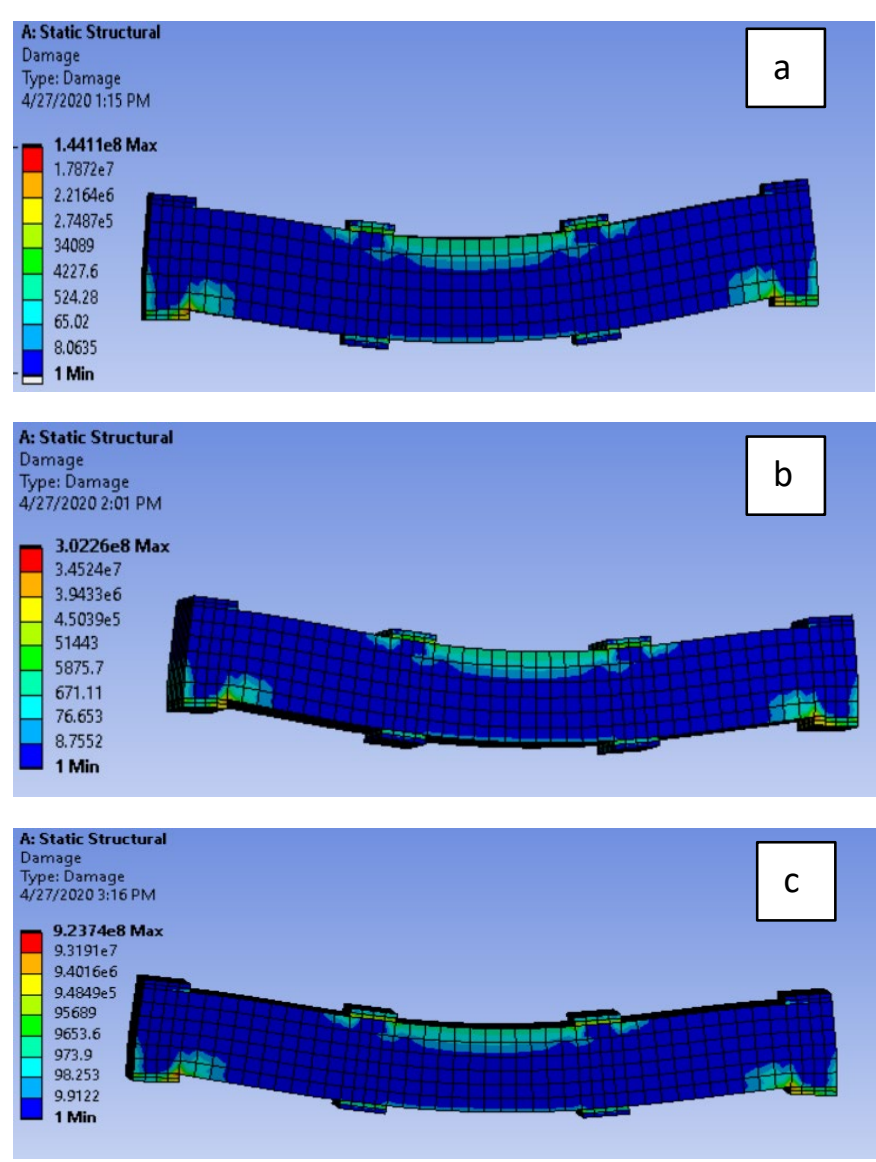

Figure 12. Fatigue Damage Analysis of 3 Different Beam Specimens

\section{Conclusions}

The utilization of FEA, results in simplifying the problem and makes the process easier. The laboratory-related analysis can be minimized and utilized only for calibration and material specification. This study provides some valuable insight and understanding of the FEA in the modeling of asphalt materials. Furthermore, the study results in some of the useful observations in the determination of total deformation, stress, and strain in various cases. The fatigue-related analysis was also an essential aspect in the determination of life and damage of the asphalt beam. Three different cases of beam specimens were analyzed for knowing the performance of the beam.

Those three different specimens of the beam showed a different kind of life and damage performance related to fatigue. The beam with higher stiffness had a significantly higher minimum critical life, which gradually decreased with lower stiffness. In the case of damage analysis, an opposite trend of performance was observed. The damage was relatively quick, with increasing stiffness.

Therefore, a detailed material-oriented preliminary test is required for the design of pavement materials concerning their performance. Similarly, the FEA approach was found to be worthy in the analysis of the beam, and the performance can be quickly evaluated. A robust analysis with full function exploration in ANSYS can be the most effective way of obtaining early pavement performance through simulated studies.

\section{Limitations and Further Studies}

This study presents the case of finite element application to asphalt materials. A four-point bending test of the beam is utilized to observe the performance of asphalt material. The modeling and analysis were performed following various literature reviews, and the results were solely based on the characteristics behavior provided. Therefore, more vigorous experimental tests are much needed to validate and verify the model. The inclusion of appropriate calibration factors may result in refined values. Similarly, this study is a pathway for running the finite element simulation on asphalt materials, and the results can be utilized in the various failure-related analysis as further applications.

\section{Declaration of Conflict of Interests}

The authors declare that there is no conflict of interest.

\section{References}

[1.] Souliman, M., "Integrated Predictive Model for Healing and Fatigue for Asphalt Concrete," Arizona State University (2012).

[2.] Witczak, M., Mamlouk, M., Souliman, M., and Zeiada, W. "Laboratory Validation of an Endurance Limit for Asphalt Pavements," Transportation Research Board, Washington D.C. (2013).

[3.] Sukumaran, B., Willis, M., and Chamala, N., "Three Dimensional Finite Element Modeling of Flexible Pavements," Advances in Pavement Engineering (2005)

[4.] Erkens, S., Liu X., and Scarpas, A., "3D Finite Element Model for Asphalt Concrete Response Simulation," International Journal of Geo-mechanics (2006).

[5.] Hadi M. N. and Bodhinayake, B., "Non-linear finite element analysis of flexible pavements," Advances in Engineering Software (2003).

[6.] Pengfei, L., Wang, D., Otto F., Hu, J., and Oeser, M., "Application of semi-analytical finite element method to evaluate asphalt pavement bearing capacity," International Journal of Pavement Engineering (2015).

[7.] Nilaward, T., Shih, C., White, T., and Ting, E., "Three-Dimensional Finite Element Program for Pavement Analysis, FHWA/IN/JHRP96/21," Indiana Department of Transportation, Indianapolis (1998).

[8.] Mulungye, R., Owen, P., and Mellon, K., "Analysis of Response of Flexible Pavements Using Finite Element Method," The ITB Journal (2005).

[9.] Vijapure, P., Shurpali, P., Agarwal, P., and Allapur, S., "Finite Element Analysis of Flexible Pavement," International Journal of Computer \& Mathematical Scie (2015).

[10.] Adhikari S. and You, Z. "Fatigue Evaluation of Asphalt Pavement using Beam Fatigue Apparatus," The Technology Interface Journal (2010).

[11.] Coni, M., Portas, S., Isola, R., and Oliveira, J., "FE evaluation of 4point bending test for fatigue cracking assessment". (2008)

[12.] Huurman M., and Pronk, A., "A detailed FEM simulation of a 4point bending test device," in 4PB Workshop, Davis (2012).

[13.] Caserio, A. K., MSC Nastran Embedded Fatigue User's Guide, MSC Software (2019). 
[14.] "What is Fatigue Analysis," 0204 2020. [Online]. Available: https://simulatemore.mscsoftware.com/what-is-fatigueanalysis-msc-nastran/.

\section{How to Cite This Article}

Nitish R. Bastola, Mena I. Souliman, and Zabi Ahmed Mohammed, Preliminary Finite Element Modeling of Asphalt Material Using ANSYS, Civil Engineering Beyond Limits, 1(2021), 21-27.

https://doi.org/10.36937/cebel.2021.001.005 